To the Editors:

\title{
Screening for diabetes mellitus in pregnancy
}

We read with interest the research letter on the above subject which appeared in the Ceylon Medical Journal 2002; 47: 38-39, and like to clarify certain points.

Gestational diabetes mellitus (GDM) is a well known entity which is diagnosed on $75 \mathrm{~g}$ oral glucose tolerance test and is a defined blood glucose criteria [1]. The other abnormality of carbohydrate metabolism which occurs during pregnancy is impaired glucose tolerance which has almost the same impact as GDM [2].

We do not agree with their statement, "In high risk populations such as Asians, the World Health Organization (WHO) advocates screening during the first trimester to detect previously undiagnosed diabetes and a formal oral glucose tolerance test between 24 and 28 weeks of gestation to diagnose gestational diabetes mellitus". One of the principal criteria for screening as defined by the WHO is that the test should be applicable to the whole population. Universal screening for GDM is superior to risk factor based screening, in detecting more cases, facilitating early diagnosis and ensuring improved pregnancy outcome [3].

Two types of screening tests are being done in most of the specialised antenatal clinics worldwide to detect GDM. They are the 2-hour post-prandial blood glucose and the $50 \mathrm{~g}$ 1-hour glucose challenge test. When interpreting the $50 \mathrm{~g}$ 1-hour glucose challenge test the threshold for further testing may be chosen based on the goal of the screening program, either to maximise sensitivity at the expense of more diagnostic testing by using a $130 \mathrm{mg} / \mathrm{dL}$ cutoff or to increase specificity at the cost of some sensitivity by using a $140 \mathrm{mg} / \mathrm{dL}$ cutoff [4]. Ideally the screening test should be done at 16 weeks and repeated in the third trimester to identify late onset of GDM and a positive test should be followed by a $75 \mathrm{~g}$ oral glucose tolerance test. We are now doing research to find out which screening test is better to detect GDM in our population.

\section{References}

1. Coustan DR. Screening and diagnosis of gestational diabetes. Baillieres Clinical Obstetrics and Gynaecology 1991; 5: 293-313.

2. Aberg A, Rydhstroem H, Frid A. Impaired glucose tolerance associated with adverse pregnancy outcome: a population-based study in southern Sweden. American Journal of Obstetrics and Gynaecology 2001; 184: 77-83.

3. Griffin ME, Coffey M, Johnson H, Scanlon P, Foley M, Stonge J, et al. Universal vs. risk factor based screening for gestational diabetes mellitus: detection rates, gestation at diagnosis and outcome. Diabetic Medicine: Journal of the British Diabetic Association 2000; 17: 26-32.

4. Coustan DR. Methods of screening for and diagnosing of gestational diabetes. Clinics in Perinatology 1993; 20: 593-602.

Hiran Samarage, Senior Lecturer and Obstetrician and Gynaecologist, and Sanjeewa Padumadasa, Registrar, Professorial Obstetrics and Gynaecology Unit, Teaching Hospital, Galle, Sri Lanka. 